\title{
Phase stable net acceleration of electrons from a two-stage optical accelerator
}

\author{
Christopher M. S. Sears, ${ }^{1,2, *}$ Eric Colby, ${ }^{1}$ R. J. England, ${ }^{1}$ Rasmus Ischebeck, ${ }^{1}$ Christopher McGuinness, ${ }^{1}$ Janice Nelson, ${ }^{1}$ \\ Robert Noble, ${ }^{1}$ Robert H. Siemann, ${ }^{1}$ James Spencer, ${ }^{1}$ Dieter Walz, ${ }^{1}$ Tomas Plettner, ${ }^{3}$ and Robert L. Byer ${ }^{3}$ \\ ${ }^{1}$ Stanford Linear Accelerator Center, Menlo Park, California 94025, USA \\ ${ }^{2}$ Max-Planck-Institut für Quantenoptik, Hans-Kopfermann-Strasse 1, D-85748 Garching, Germany \\ ${ }^{3}$ Stanford University, Stanford, California 94305, USA \\ (Received 4 September 2008; published 21 October 2008)
}

\begin{abstract}
In this article we demonstrate the net acceleration of relativistic electrons using a direct, in-vacuum interaction with a laser. In the experiment, an electron beam from a conventional accelerator is first energy modulated at optical frequencies in an inverse-free-electron-laser and bunched in a chicane. This is followed by a second stage optical accelerator to obtain net acceleration. The optical phase between accelerator stages is monitored and controlled in order to scan the accelerating phase and observe net acceleration and deceleration. Phase jitter measurements indicate control of the phase to $\sim 13^{\circ}$ allowing for stable net acceleration of electrons with lasers.
\end{abstract}

DOI: 10.1103/PhysRevSTAB.11.101301

PACS numbers: 41.75.Jv, 41.60.Cr, 41.85.Ct

Shortly after the development of Q-switched lasers, methods for electron acceleration using lasers began to be explored [1]. Since then several experimental demonstrations of a direct electron-laser interaction have occurred [2-4]. These first experiments utilized an electron beam that was much longer than the laser period. Electrons were present at all phases of the accelerating field and thus an energy modulation occurred rather than net acceleration. In an experiment by Kimura et al. [5], net acceleration from a laser interaction was obtained by slicing the electron beam into many microbunches spaced at the optical period. This microbunching is done by first energy modulating a long electron beam in a short optical accelerator and then passing the beam through an energy dispersive chicane to form the microbunches. The microbunched beam is then accelerated in a second optical accelerator.

One of the primary challenges presented by staged optical acceleration is the measurement and control of the electron phase relative to the laser field. At optical frequencies, acoustic vibrations can easily disrupt the phase in a multistaged accelerator. In this article we present a staged optical acceleration experiment in which care has been taken to measure and control the optical phase between stages. The phase is then scanned in a controlled way to observe both accelerating and decelerating phases.

The production of a microbunched beam spaced at $800 \mathrm{~nm}$ was recently demonstrated [6] using the same method as Kimura et al. [5]. The microbunch structure was characterized with coherent optical transition radiation and found to show good agreement with a 1D analytic theory. The density modulation $\rho(z)$ can be expressed $[7,8]$ as a Fourier series whose coefficients are Bessel functions depending on the amount of dispersion $\left(R_{56}\right)$ and the modulation from the inverse-free-electron-laser (IFEL)

*cmsears@mpq.mpg.de
( $\eta$ ) by

$$
\rho(z)=\rho_{0}\left[1+2 \sum_{n=1}^{\infty} b_{n} \cos \left(n k_{l} z\right)\right]
$$

where

$$
b_{n}=J_{n}\left(n k_{l} R_{56} \frac{\eta}{\gamma_{0}}\right) \exp \left[-\frac{n^{2}}{2}\left(k_{l} R_{56} \frac{\sigma_{\gamma}}{\gamma_{0}}\right)^{2}\right],
$$

$\rho_{0}$ is the initial charge density entering the IFEL, $\gamma_{0}$ and $\sigma_{\gamma}$ are the initial mean electron energy and energy spread, and $k_{l}=2 \pi / \lambda_{l}$ is the laser wave number. The exponential term in the expansion coefficients defines the washout of the bunching due to the initial energy spread.

In a short second stage accelerator, in which changes in longitudinal position due to velocity differences or betatron motion are negligible, the net energy shift of the entire electron distribution is

$$
\begin{gathered}
\Delta E=\frac{1}{\lambda_{l} \rho_{0}} \int_{0}^{\lambda_{l}} \rho(z) A \cos \left(k_{l} z+\phi\right) d z, \\
\Delta E=A b_{1} \cos \phi,
\end{gathered}
$$

where $A$ is the amplitude of peak acceleration in the second stage. Notice that the total energy shift is related only to the first term of the Fourier series expression for the microbunching. On the other hand, maintaining a small final energy spread of the accelerated microbunches would require optimizing the higher terms in the Fourier series.

Although the main interest in the experiment is observing the shift of the total energy of the beam, the second interaction also affects the total energy spread and asymmetry of the beam energy distribution. Figure 1 shows a Monte Carlo simulation of the progression of the electron phase space and energy spectrum for a single slice of optical phase. After modulation in the IFEL, microbunches 

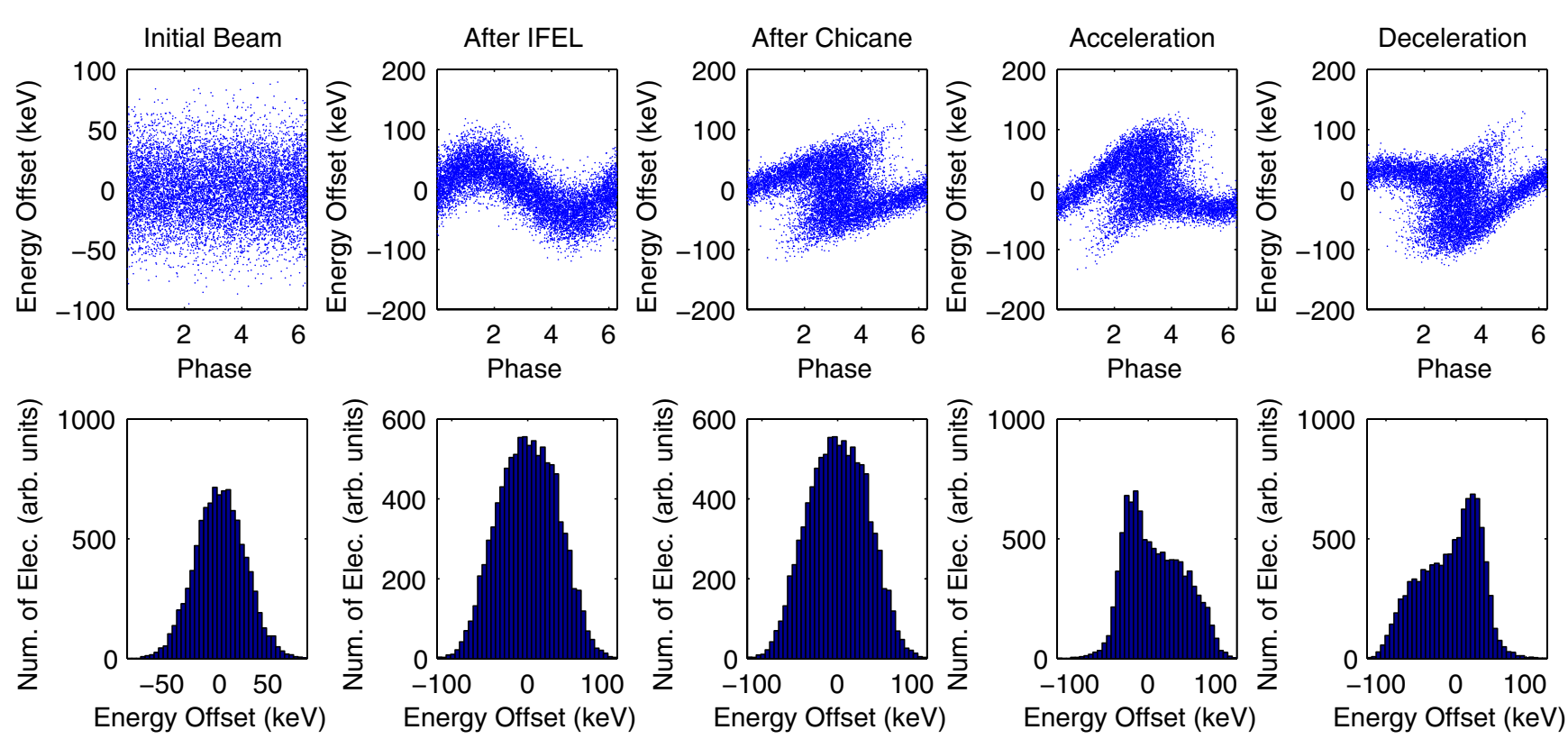

FIG. 1. (Color) Monte Carlo simulation of the evolution of a microbunch in the net acceleration experiment. Shown are the longitudinal phase space (top row) and energy spectrum (bottom row) starting before the IFEL and finishing with accelerating and decelerating cases for the ITR interaction.

are formed in the chicane with the microbunches located at a phase of $\pi / 2$ from the peak modulation in the IFEL. Thus, peak net acceleration is obtained in the second stage when the second stage is at a phase of $\pi / 2$ relative to the first. The simulations also show variation in the energy spectrum asymmetry, defined as asym $=\left(\sigma_{\text {uwhm }}-\right.$ $\left.\sigma_{\text {lwhm }}\right) / \sigma_{\text {fwhm }}$, where $\sigma_{\text {uwhm }}, \sigma_{\text {lwhm }}$, and $\sigma_{\text {fwhm }}$ are the upper, lower, and full widths at half-maximum with the center point for the half-widths defined as the value for peak intensity in the spectrum. As can be seen from the energy spectra, the spectral asymmetry changes as a function of phase along with the mean energy. Comparison of the energy spectra to the longitudinal phase space reveals that the asymmetry variation is caused by flattening in energy of an unbunched portion. For the accelerating case, this portion is at lower energy and for the decelerating case, at higher energy. Thus, the asymmetry variation is $\pi$ out of phase relative to the net acceleration.

Using a collection of simulations like those plotted in Fig. 1 for different phases between the first and second stages, one can also see that the energy spread also varies sinusoidally with an offset of $-\pi / 2$ relative to the net energy of the beam. At a phase of $+\pi / 2$, the second stage essentially "undoes" the modulation imparted in the first. This is not perfect because the dispersion imparted by the chicane distorts the initially sinusoidal energy modulation.

This research was conducted at the NLCTA facility at SLAC, a $60 \mathrm{MeV}$ X-band accelerator that was recently upgraded with an S-band photoinjector for laser acceleration research. The NLCTA beam line includes a chicane for energy collimation. Using the energy collimation, the accelerator produces $1 \mathrm{pC}, \sim 1 \mathrm{ps}$ pulses with $\leq 0.05 \%$ energy spread. The laser system used to produce UV for the photoinjector also provides IR pulses for laser acceleration research. This setup gives the timing stability necessary for the experiments [9]. Table I lists the parameters of the net acceleration experiment.

The second stage accelerator uses the inverse transition radiation (ITR) mechanism [10]. This mechanism was used as it had previously been developed and demonstrated in recent work $[10,11]$. In the ITR accelerator, the electron beam and laser are incident on a thin metal boundary. The laser propagates at an angle of $1 / \gamma$ with respect to the electrons. The laser and electrons interact in the space upstream of the boundary with the interaction terminating at the boundary allowing for net acceleration.

Although using the ITR mechanism in an initial demonstration experiment has the advantage of preexisting hardware and experience, its use does also bring a few disadvantages. The first is the small interaction strength. With the available laser power, the ITR mechanism can impart $\sim 30 \mathrm{keV}$ of peak acceleration. This is comparable to the initial energy spread of the beam and less than the total energy spread of the beam after the laser interactions. This leads to an even smaller shift in the total energy of the beam in the staged acceleration experiment since not all electrons experience the peak acceleration and some, even with microbunching, are decelerated. The second disadvantage comes from the geometry of the laser-electron interaction. Since the laser and electron intercept at an angle of $\sim 1 / \gamma$, for a given cross section of the electron beam, there exists different areas of laser phase across the horizontal direction of the beam. These fringes are spaced by $\lambda \gamma=94 \mu \mathrm{m}$. In order to observe net acceleration, it is 
TABLE I. Experimental parameters for the net acceleration experiment. All width values are fwhm.

\begin{tabular}{lc}
\hline \hline Parameter & Value \\
\hline Ebeam energy & $60 \mathrm{MeV}$ \\
Energy spread & $45 \mathrm{keV}$ (typical) \\
Ebeam pulse length & $0.5 \mathrm{ps}$ (typical) \\
Ebeam spot size & $100 \mu \mathrm{m}$ (nominal) \\
Laser pulse length & $0.75 \mathrm{ps}$ (nominal) \\
Laser energy & $1 \mathrm{~mJ} / \mathrm{pls}$ \\
IFEL/ITR laser split ratio & $35 / 65$ \\
IFEL laser spot size & $500 \mu \mathrm{m}$ \\
ITR laser spot size & $200 \mu \mathrm{m}$ \\
Undulator period & $1.8 \mathrm{~cm}$ \\
Number of periods & 3 \\
Undulator strength $\left(a_{w}\right)$ & 0.46 \\
Chicane $R_{56}$ & $0.06-0.22 \mathrm{~mm}$ \\
ITR crossing angle & $8 \mathrm{mrad}$ \\
\hline \hline
\end{tabular}

therefore necessary to restrict the transverse size of the electron beam to less than half a fringe. This is done using a tungsten slit. Electrons that do not pass through the slit are scattered on the tungsten and do not reach the energy spectrometer.

The net acceleration experiment builds on the hardware for the microbunching experiment [6]. Figure 2 shows the layout for the experiment. The laser light is split to drive the two stages with each path having separate focusing optics and remote controllable steering mirrors. In the arm driving the second stage there is also a delay arm to obtain temporal coincidence in the two laser-electron interactions and a piezo mounted mirror for scanning the timing on the optical scale. Inside the vacuum chamber are the undulator for the IFEL interaction, the chicane, and the second stage ITR accelerator.

Arguably the key technical challenge in this experiment is the measure and control of the optical phase between the laser-electron interaction stages. The layout is essentially a large interferometer, with the electron beam serving to communicate the phase between the IFEL and the second stage accelerator. To find the phase between the IFEL and ITR paths, a second laser path is installed that closely follows the $800 \mathrm{~nm}$ drive laser. Prior to entering the main chamber containing the accelerators, the monitor laser is brought out and recombined with the other arm of the interferometer. The interference pattern is observed with a CCD and used to interpret the phase of the drive laser in the interferometer.

Since the phase of the actual drive laser is not directly measured during the experiments, it is important to verify that the phase monitor system tracks the actual phase of the drive laser. To do this, prior to beam experiments, another CCD was temporarily placed inside the experiment chamber to observe interference fringes from the IFEL and ITR lasers. Comparisons were made between the phase of the $800 \mathrm{~nm}$ light to that of the phase monitor.

Each of the two interferometers were found to have a fast uncorrelated jitter of around $13^{\circ}$. When the phase was actively scanned using the piezo mirror [Fig. 3(a)], the phase between the two monitors tracked well. Over very long time scales, however, the phase between the two interferometers would wander [Fig. 3(b)]. In this case the slow drift was more than $2 \pi$ during the course of an hour. Fortunately, this is much longer than the typical data set which are acquired in a few minutes. In addition, it was found during postanalysis that the data itself can be used to account for the slow drift.

Prior to net acceleration, the IFEL and ITR interactions are first observed individually using the cross-correlation technique described in [6]. The coarse delay between the IFEL and ITR optical paths is adjusted so that the two interactions occur with the same laser timing. Both the IFEL and ITR interactions are then used together with a fixed laser to electron delay to obtain net acceleration. The optical phase offset between the two stages is scanned using the piezo driven mirror producing phase correlated

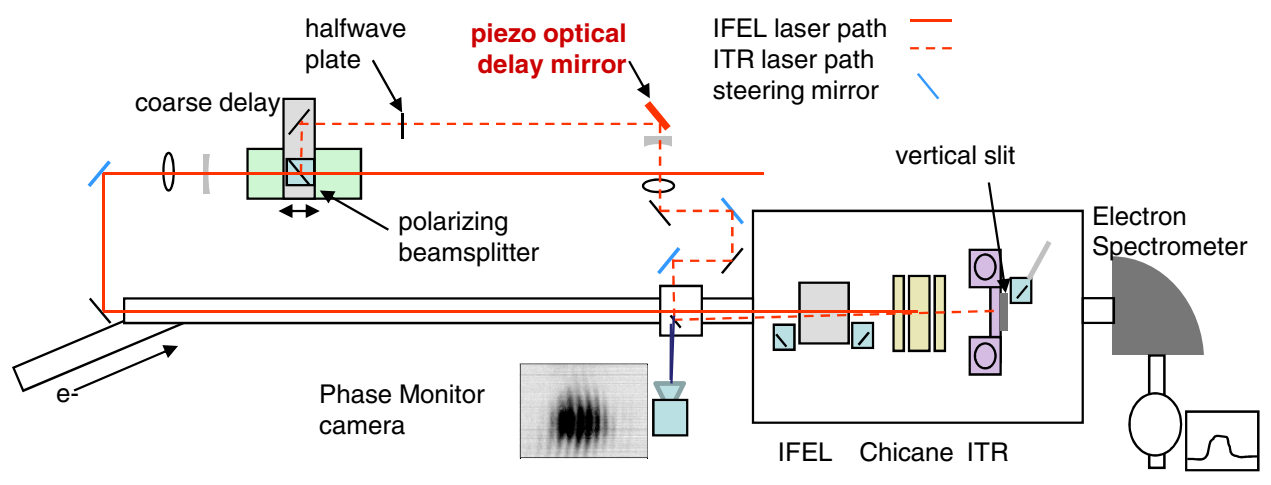

FIG. 2. (Color) Layout of the net acceleration experiment. The laser is split at a polarizing beamsplitting cube to drive both the ITR acceleration and the IFEL. The ITR path includes a piezo driven mirror for varying the optical phase. Each arm is $\sim 5 \mathrm{~m}$ in path length. Interferometric noise measurements indicate the optical paths are stable to within $\sim 20 \mathrm{~nm}$ or 2 parts per billion of the total path. 

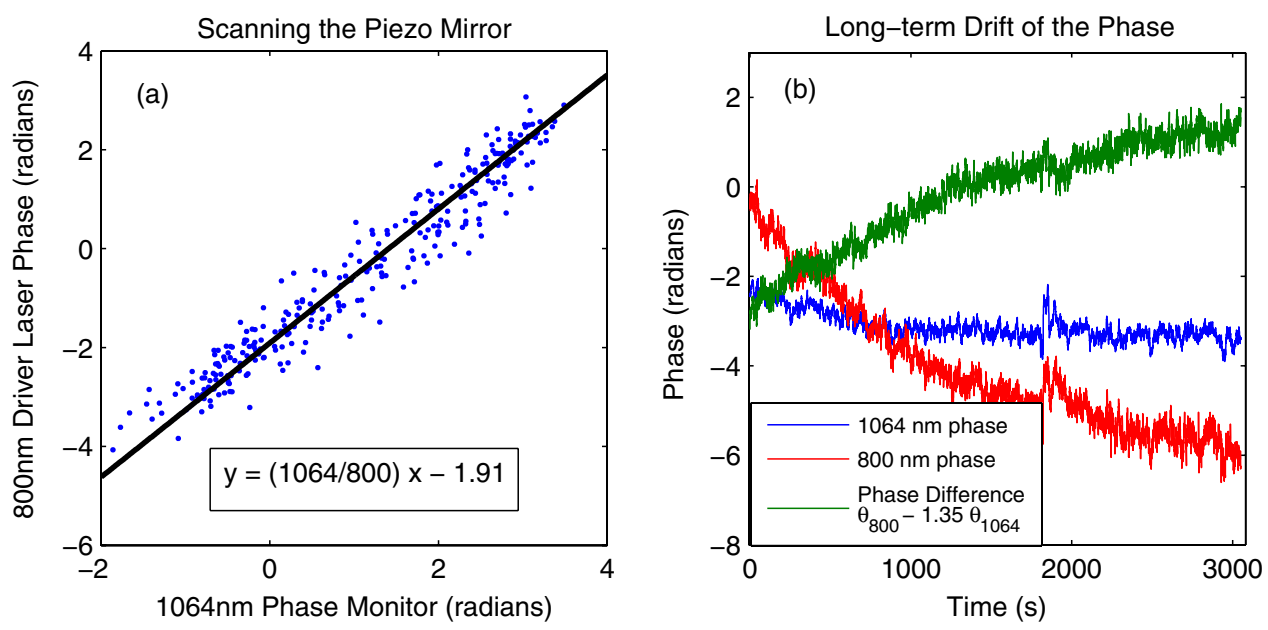

FIG. 3. (Color) Phase stability of the net acceleration experiment. The figure on the left shows the linearity of the phase monitor to the experiment laser phase while the phase is actively scanned using the piezo delay mirror. When the phase is fixed the slow drift of the phase can be observed. Over time the experiment laser phase can wander away from the phase of the monitor system. The fast jitter is $13^{\circ} \mathrm{rms}$.

oscillations in the electron spectrum centroid energy, energy spread, and asymmetry.

One of the challenges to observing net acceleration and the expected sinusoidal variation of the acceleration with IFEL-ITR phase is the small laser-electron interaction strength compared to the noise. In the end the measured net acceleration was comparable to the mean energy and energy spread jitters and much less than the total energy spread. To help observe the acceleration, a first method employed to analyze the data uses a frequency "lock-in" method for obtaining small signals in a noisy background. The piezo is driven linearly to obtain $2 \pi$ of laser phase shift every 10 seconds, or 100 events at $10 \mathrm{~Hz}$. Since the piezo has a finite range, the motion is actually a saw-tooth function. In postanalysis the electron energy spectra quantities are Fourier transformed into the frequency domain. Figure 4 shows the discrete Fourier transform for the phase monitor signal along with the electron spectra centroid, spread, and asymmetry. The phase monitor duplicates the original saw-tooth drive function to the piezo. Clearly visible in the remaining three plots is a signal at the original drive frequency of $0.1 \mathrm{~Hz}$.

The analysis of Fig. 4 confirms that net acceleration is taking place and that all three electron energy quantities vary sinusoidally with the phase as expected. However, interpreting the amplitude of net acceleration from this analysis is less clear. In addition, a more compelling demonstration of the net acceleration would be made by direct correlation between the IFEL-ITR phase and the energy centroid. This, however, runs into difficulties regarding poor signal to noise, especially for discerning the energy centroid shift. In the case of the energy spread variation, the signal to noise is much better and the sinusoidal variation can be seen in binned correlation plots using only a few hundred laser-electron interaction events. Observing the other quantities requires greater statistics. However, for long runs the possibility of the drift of the phase offset between the laser-electron interaction and the monitor system could potentially washout a correlation signal. Figure 5 shows an example of this drift seen in a long data scan of 7000 events taken over a 12 min period. Two subsets of the data taken minutes apart show differing phase offset in the energy spread to phase correlation plot. Both sets clearly show the sinusoidal variation but with much different offsets. If the two subsets were combined, the variation would disappear. Thus, in order to take advantage of greater statistics to clearly distinguish the oscillation of the energy centroid, it is necessary to account for the drift of the phase offset. By taking several subsets the time evolution of the phase offset can be found from the energy spread data. This evolution is shown in the right plot of Fig. 5.

The phase drift observed in Fig. 5 can be used to produce drift corrected phase correlations for the entire data set. This is shown in Fig. 6. The data for the three correlation plots are binned and plotted along with a fit to a cos function. Cuts are applied to remove laser off and "bucket-hop" events where no laser-electron interaction occurs. The error bars are the standard deviations of the means for each bin. With the benefit of greater event statistics and slow phase drift correction, the sinusoidal variations of all three energy spectra quantities are clear. As predicted from the simulation, the asymmetry is $\sim \pi$ out of phase with respect to the centroid while the energy width is $\sim-\pi / 2$ out of phase. Prior to this run, the IFEL and ITR interactions were established separately with modulation strengths of 80 and $35 \mathrm{keV}$, respectively, and an initial energy spread of $47 \mathrm{keV}$ fwhm without laser interactions. This gives an average bunching factor for the beam of $b_{1}=0.35$ [Eq. (1)] and an expected centroid 

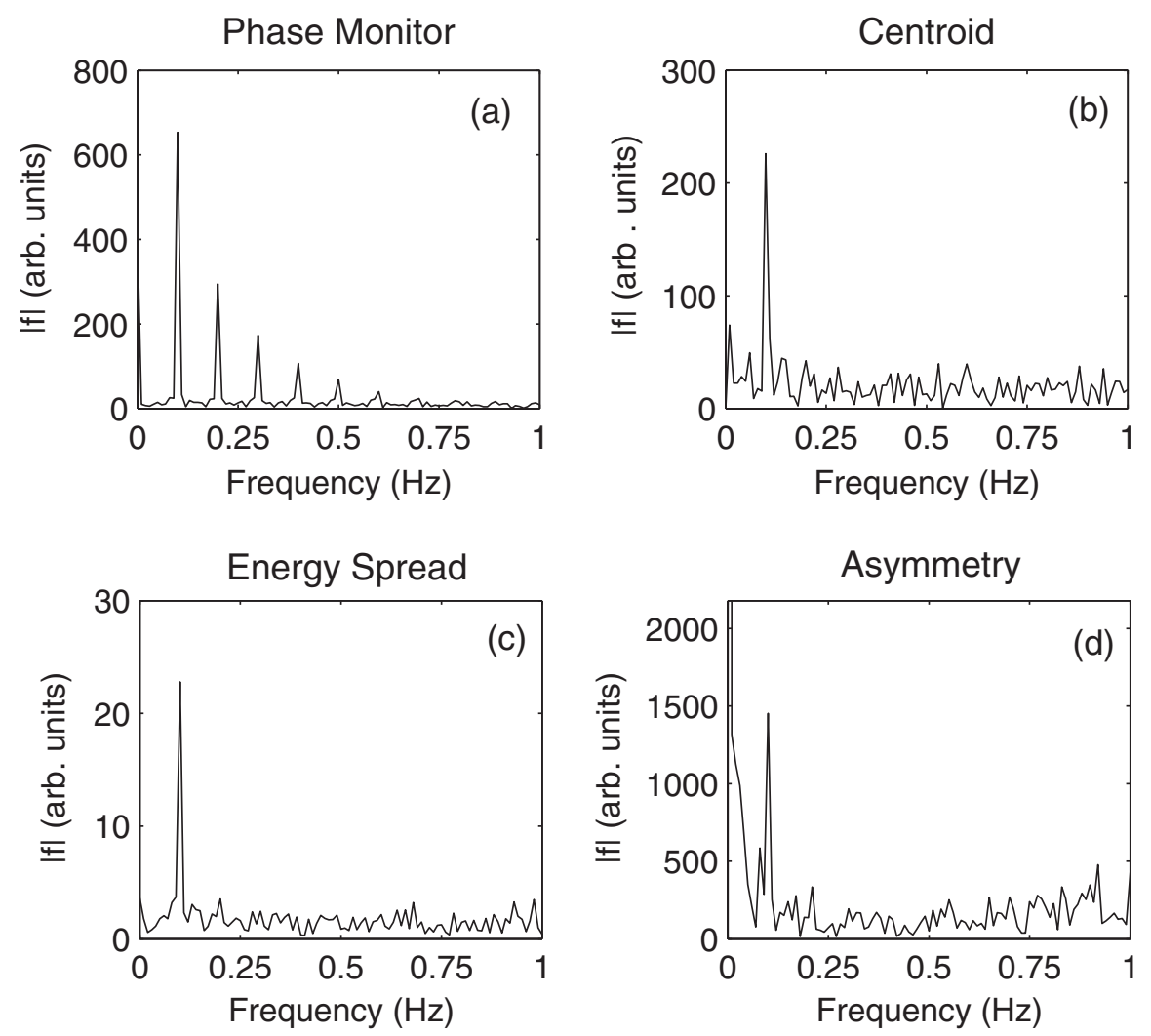

FIG. 4. Frequency domain analysis of net acceleration experiment. The phase is scanned with a saw-tooth function as seen by the phase monitor (a). The electron centroid energy (b), energy spread (c), and asymmetry (d) show variation at the drive frequency of $0.1 \mathrm{~Hz}$, confirming that net acceleration is occurring in the staged experiment.

shift of $b_{1} \times 35 / 2=6 \mathrm{keV}$. This compares to a measured amplitude of just $1.2 \mathrm{keV}$.

Many attempts were made to investigate the smaller than expected energy shift. One possible source for the short fall is misalignment of the phase fronts of the microbunched electrons compared to the laser in the second stage. Despite the total measured centroid shift falling short of the ex- pected signal, the experiment did succeed in measuring net acceleration and controlling the acceleration phase. This is the first demonstration of direct optical acceleration at visible wavelengths. The experiment explored many of the important aspects of direct laser acceleration including staging of two optical accelerators. A key aspect of this experiment was the optical phase monitoring and control.
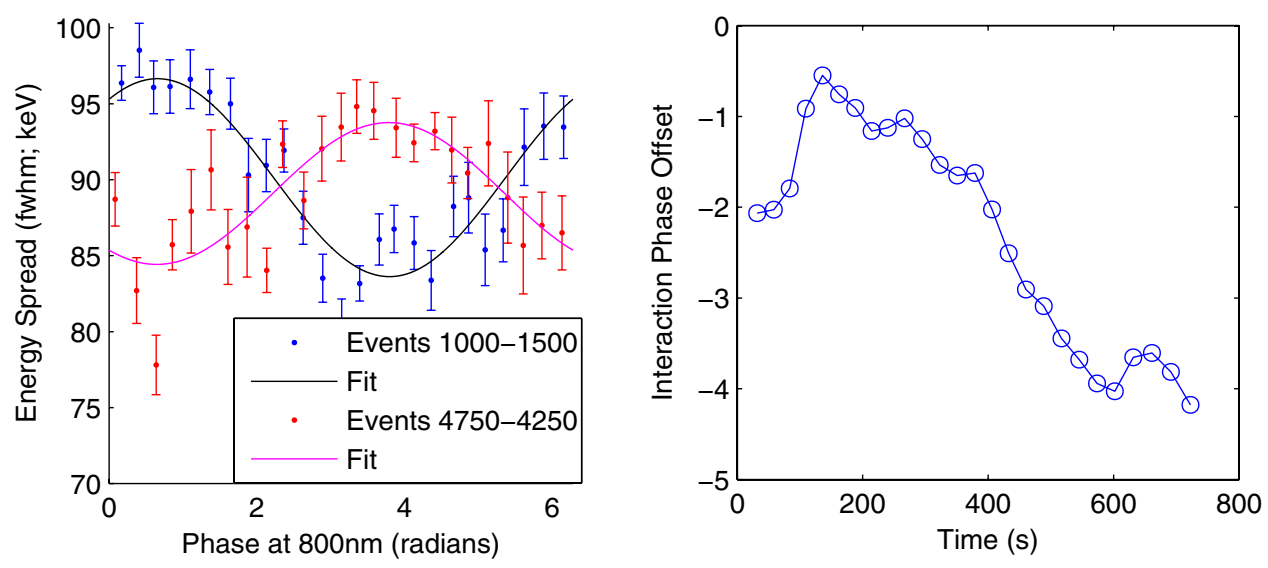

FIG. 5. (Color) Drift of the phase offset between the acceleration phase versus the monitor over time. Left: Two short subsets of a long run show differing phase offset in the energy spread versus phase correlation plot. Right: The phase offset at each subset of a long data scan shows the time evolution of the offset during the full data scan. 

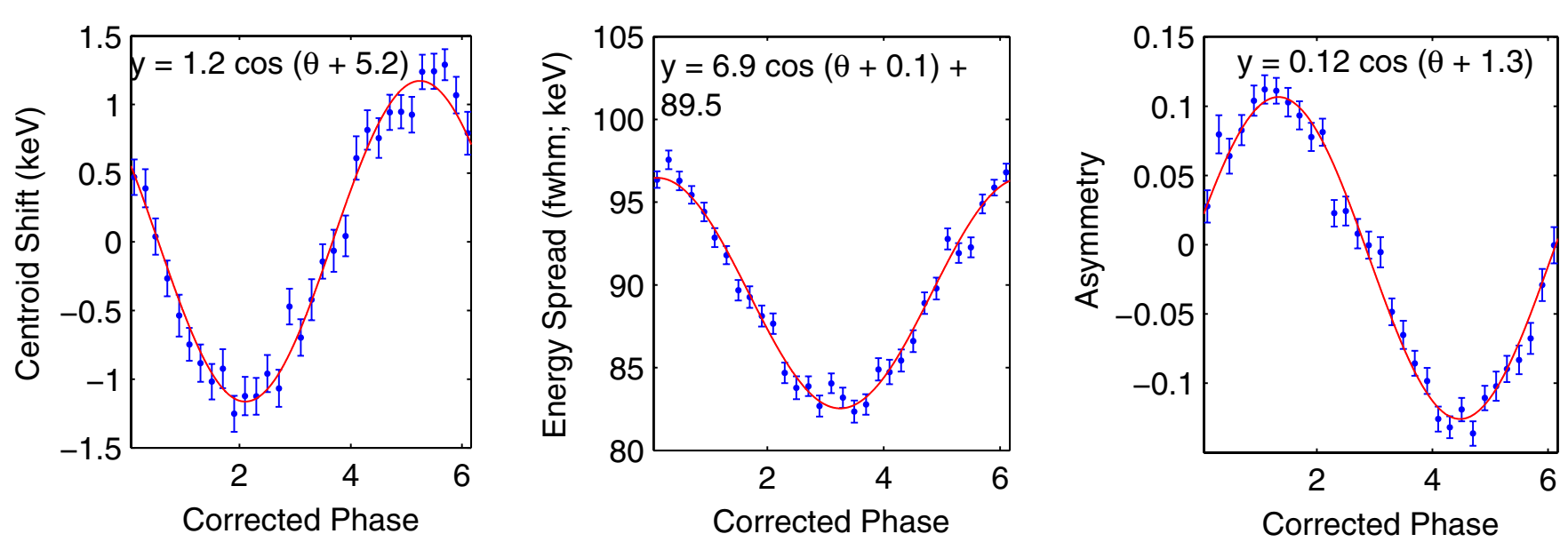

FIG. 6. (Color) Correlation of electron spectrum centroid energy (left), energy spread (center), and asymmetry (right) with the corrected phase subtracting slow drift. The error bars are the deviations of the means for the binned data. Also shown are sinusoidal fits to each set.

Future experiments with a stronger second stage interaction will be needed to further explore issues such as charge capture. This might be provided by using photonic band gap structures [12] to build optical scale dielectric waveguides for guiding the laser [13]. Such a device could sustain large accelerating gradients approaching $1 \mathrm{GeV} / \mathrm{m}$ while at the same time requiring less pulse energy due to the small transverse dimensions leading to good coupling efficiency [14]. The first experiments with such devices are currently being planned.

\section{ACKNOWLEDGMENTS}

The authors wish to acknowledge the efforts and contributions of the NLCTA Operations group: Justin May, Doug McCormick, Tonee Smith, Richard Swent, and Keith Jobe. We would also like to thank Walt Zacherl and Bruce Rohrbough for their work on laser diagnostics. This work is supported by Department of Energy Contracts No. DEAC02-76SF00515 and No. DE-FG02-03ER41276.

[1] M. J. Feldman and R. Y. Chiao, Phys. Rev. A 4, 352 (1971).

[2] W. D. Kimura, G. H. Kim, R. D. Romea, L. C. Steinhauer, I. V. Pogorelsky, K. P. Kusche, R. C. Fernow, X. Wang, and Y. Liu, Phys. Rev. Lett. 74, 546 (1995).
[3] C. M. S. Sears, E. R. Colby, B. M. Cowan, R. H. Siemann, J. E. Spencer, R. L. Byer, and T. Plettner, Phys. Rev. Lett. 95, 194801 (2005).

[4] P. Musumeci et al., Phys. Rev. Lett. 94, 154801 (2005).

[5] W. D. Kimura et al., Phys. Rev. Lett. 86, 4041 (2001).

[6] C.M.S. Sears et al., Phys. Rev. ST Accel. Beams 11, 061301 (2008).

[7] S. Baccaro, F. DeMartini, and A. Ghigo, Opt. Lett. 7, 174 (1982).

[8] A. Luccio, G. Matone, L. Miceli, and G. Giordano, Laser Part. Beams 8, 383 (1990).

[9] C. McGuinness, R. Byer, E. Colby, R. Ischebeck, R. Noble, T. Plettner, C. M.S. Sears, R. Siemann, J. Spencer, and D. Walz, in Proceedings of the 2007 Particle Accelerator Conference, Albuquerque, New Mexico, 2007 (IEEE, Albuquerque, New Mexico, 2007), p. 4195.

[10] T. Plettner, R. L. Byer, E. Colby, B. Cowan, C. M. S. Sears, J.E. Spencer, and R. H. Siemann, Phys. Rev. Lett. 95, 134801 (2005).

[11] T. Plettner, R. L. Byer, E. Colby, B. Cowan, C. M. S. Sears, J. E. Spencer, and R.H. Siemann, Phys. Rev. ST Accel. Beams 8, 121301 (2005).

[12] J. D. Joannopoulos, R. D. Meade, and J. N. Winn, Photonic Crystals: Molding the Flow of Light (Princeton University Press, Princeton, New Jersey, 1995), p. 08540.

[13] X. E. Lin, Phys. Rev. ST Accel. Beams 4, 051301 (2001).

[14] Y. C. N. Na, R. H. Siemann, and R. L. Byer, Phys. Rev. ST Accel. Beams 8, 031301 (2005). 